\title{
KEEPING TRACK OF PRODUCTIVITY IN HOUSE-BUILDING
}

\author{
Alexander Jimenez ${ }^{1}$, Torbjörn Ilar², and Lars Stehn³
}

\begin{abstract}
The broad nature of productivity leads to different interpretations and uses depending on the purpose, context and levels giving many ways for measuring productivity. However, measuring productivity in ways that reveal substantial information for operational improvements in house-building seems challenging, and methods like single-factor productivity are reported not to be common in systematic ways to that end. It is unclear how productivity is used by house-builders on-site to control, evaluate and improve operations. This study addresses how Swedish house-building companies keep track of productivity in the processes and sub-processes of production. Productivity measurements and the control of influencing factors on productivity are discussed with findings from interviews and workshops with representatives from five Swedish housebuilding companies. Results show that some productivity measurements are done in parallel with "ordinary" project management assessments to control time and cost. Yet, most factors seemingly affecting productivity are measured qualitatively and "stay in the mind" of the managers. However, some results indicate an increased understanding amongst managers for using performance indicators to track and develop activities and processes assisted with digital tools. Still, further investigation is needed to better understand how factors influencing productivity can systematically be assessed and incorporated into methods for operational development.
\end{abstract}

\section{KEYWORDS}

Productivity measurements, house-building, work flow, process, daily huddle

\section{INTRODUCTION}

The call for improved productivity is a common statement in construction. Value added productivity statistics derived from a global survey of businesses presented by McKinsey consultants (Barbosa et al. 2017), reveal that construction is lagging behind other industries. However, to measure productivity in ways that allow for operational improvements is a challenge in on-site house-building (HB) (Motwani et al. 1995; Crawford and Vogl 2006). Firstly, the broad concept of productivity is understood and used with differently depending on the purpose and context leading to many ways for

$1 \quad$ PhD Student, Construction Management \& Building Technology, Dept. of Civil, Environmental \& Natural Resource Engineering, Luleå Univ. of Technology, Sweden, alexander.jimenez@,1tu.se, orcid.org/0000-0001-7382-6936

2 Sen. Lecturer, Manufacturing Systems engineering, Dept. of Engineering Sciences \& Mathematics, Luleå Univ. of Technology, Sweden, torbjorn.ilar@1tu.se, orcid.org/0000-0002-3007-9560

3 Prof. Construction Management \& Building Technol., Dept. of Civil, Envir. \& Natural Resource Engineering, Luleå Univ. of Technology, Sweden, lars.stehn@ltu.se, orcid.org/0000-0002-3067-9451 
defining productivity (Huang et al. 2009; Crawford and Vogl 2006). For instance, productivity can be defined as the ability to transform inputs into products (Allmon et al. 2000), but also mathematically as the relation of output over inputs (Tangen 2005). Secondly, there are different methods, e.g. single- (SFP) and multi-factor (MFP) productivity measurements to measure productivity with different purposes in different organizational levels (task, project, organization) (Huang et al. 2009). SFP, commonly used by economics on the national level, receives critique from industry practitioners (IPs). For instance, IPs are reported (Jimenez et al. 2019) to find little use from the value added SFP, as it rather reflects economic activity than performance of actions related to HB operations. MFP, incorporates many drivers of productivity to establish a clearer cause-and-effect relationship (Crawford and Vogl 2006), but has its challenges such as the need of large amounts of robust data.

It is not surprising that the broad nature of productivity can be interpreted in different ways. However, what is common between the interpretations is that the measurement of productivity can reflect how "well" actions, processes and resources (inputs) are combined to produce a given output (Tangen 2005); and to improve productivity, one needs to understand and act upon the factors that drive productivity (Choy and Ruwanpura 2006). In turn, acting systematically upon factors that affect productivity, together with the fact that construction projects plan and report accumulated resource allocation and use, how the time plan is followed versus the master production plan, design deviations etc., the IPs control, report and rearrange the production outcomes and pace, by e.g. using the Last Planner tool; could be compared to and complemented by, to a certain degree, measuring productivity (Choy and Ruwanpura 2006; Pérez et al. 2019).

Many previous studies have focused on identifying factors affecting productivity, or suggestions of what to measure (Hasan et al. 2018; Huang et al. 2009; Dolage and Chan 2013), and the different interpretations that are attained due to the broad nature of productivity make the understanding and use of productivity fuzzy. In a study written by Jimenez et al. (2019), the authors discussed how productivity could be understood. What remains unclear is how productivity is used by the IPs during on-site HB production to control, evaluate and improve operations. Thus, this study addresses if and then how representative $\mathrm{HB}$ companies (HBC) in Sweden keep track of productivity in the processes and sub-processes of HB production, i.e. plan, control, report and rearrange the production in terms of productivity. The paper presents findings from an ongoing $R \& D$ study, which has the purpose of identifying different productivity measures from the progress of sub-processes in HB projects from five of Sweden's biggest HBCs.

\section{WHAT IS PRODUCTIVITY?}

Productivity is important for creating cost and quality advantages for companies (Alby 1994). Thus, improving productivity should be a goal for companies to address to assure their place on the market. However, productivity is a multifaceted term which meaning varies depending on people, contexts, levels (task, project and organization) and purposes of addressing productivity (Pritchard 1995; Huang et al. 2009). Hence, to improve productivity can be problematic since the different understandings could include different aspects depending on who addresses the term. Therefore, to clarify the position taken in this paper, first we need to investigate what productivity can be understood and used as.

Firstly, production can be defined as the transformation of inputs into outputs, or the transformation of materials into products through flows that should add value to what is produced (Koskela 2000), and productivity is often understood as the relation between 
output and inputs (Tangen 2005). Moreover, productivity can also be seen as how much and how well companies produce from the resources used, which can include efficiency, effectiveness and other aspects that would affect the production of an outcome, such as morale, innovation, turnover, absenteeism, etc. (Bernolak 1997; Pritchard 1995).

Secondly, the term is often expressed using mathematically based definitions at different organizational levels to estimate productivity (Huang et al. 2009). One is a SFP expression of output over input, normally used as an econometric measure on the higher levels to estimate value added productivity, but also to assess labor productivity in projects and tasks as the value produced per labor costs or hours. Nevertheless, SFP includes only one input leaving out information about many different factors that affect the transformation of inputs into an output. There are other methods that counter the use of one input and reveal more information about the process, e.g. total factor productivity (TFP) (Vogl and Abdel-Wahab 2015). TFP intends to measure the performance of the production by incorporating several factors affecting the transformation of inputs into an output, hence requiring large amount of robust data. Therefore, the method normally used is the SFP.

However, the use of mathematically based definitions (such as value added SFP) based on economic statistics gathered on a national level or at the best on a building project level to estimate the complex productivity concept comes with the risk of not always reflecting all the effects and characteristics that represent productivity progress or even productivity as such (Tangen 2005). In fact, how productivity is typically defined from an economic point of view seems to be troublesome to embrace on the project and HB process sub-level as it might not fit the goals and purposes of addressing the term (Jimenez et al. 2019). In consequence, such measurements are reported by some IPs not to be used as a common praxis in systematic ways to improve operational progress (Jimenez et al. 2019).

\section{FACTORS INFLUENCING PRODUCTIVITY}

Hasan et al. (2018) identified factors affecting productivity from studies over the past 30 years. The most common were non-availability of materials, inadequate supervision, skill shortage, lack of proper tools/equipment, incomplete drawings and specifications, poor communication, rework, poor site layout, adverse weather conditions, and change orders.

Even if it can be discussed that performance and productivity are different concepts, where productivity is understood as the pure physical phenomenon of transformation of inputs into outputs, and performance as a phenomenon that includes profitability, productivity and other non-cost factors, they are related (Tangen 2005). Actions aimed to increase performance, if aligned with the company's or project's productivity goals, could also improve productivity. The reciprocity between performance and productivity is proposed to be influenced by the project culture (Aziz and Hafez 2013; Zou and Zillante 2015). The implication is that the project culture also is an indirect factor of productivity.

Moreover, as Hasan et al. (2018) state, the factors that influence productivity are not mutually exclusive. This means that the factors can be interrelated, which can make it difficult to define precise cause-and-effect relationships. Still, controlling factors through measurements could help in making the performance of the production process transparent and observable (Koskela 2000). Combining measurements in a systemic approach could then help organizations to better understand the complexity and dynamics in projects (Lantelme and Formoso 1999), facilitating the identification and learning of the causes that would affect productivity. 


\section{PERFORMANCE INDICATORS AS INDICATIVE PRODUCTIVITY MEASURES}

Construction projects are known for being unique with varying conditions such as environments, characteristics, and project management leading to productivity differences. The fact that factors that affect productivity are time- and context dependent together with the lack of standardized systems in the companies makes it hard to define a standard productivity measure (Park 2006; Hasan et al. 2018).

Many studies have been carried out to find measurement methods suitable for construction, and the use of performance indicators (PI) from benchmarking has been expressed as one way (Haponava and Al-Jibouri 2009). PIs receive critique such as not providing a holistic representation of a business, or that cause and effect relationships are hard to establish with only indicators, but are still considered important for controlling and improving performance and productivity (RSA 1995; Egan 1998). In fact, PIs, if chosen and used correctly, can give valuable feedback from sub-processes in processes (Beatham et al. 2004). PIs can then help employees to know how they perform and assist them in making decentralized decisions during operations (Lantelme and Formoso 1999).

Beatham et al. (2004) define three types of performance measures: key performance outcomes (KPOs), KPIs and perception measures. KPOs are lagging measures that show the results of a completed process, which can serve to make decisions for future processes. KPIs are leading measures, giving indications of the ongoing performance inside the processes, giving an opportunity to change actions to reach a desired outcome. Perception measures that can be used as both lagging and leading indicators (Beatham et al. 2004).

To succeed in using such measurements, Beatham et al. (2004) state that an understanding of when to use what type of measure is needed, and that to provide a holistic view, one has to mix both leading and lagging measures over the process. The measures also have to be part of a change action driven system, incorporated into the processes of the organization, engaging employees in the development and measurements of the measures. Moreover, the measures must be aligned with the objectives and strategies of the business in order to add value (Beatham et al. 2004).

Still, despite the potential of PIs, existing measures seem to be in limited use in building production (Vogl and Abdel-Wahab 2015). If used, Beatham et al. (2004) state that they tend to be KPOs, giving the house builders little chance to act in time.

\section{Planning: SETTING UP AND ENABLING FOR PRODUCTIVITY}

Production planning generally consists of setting goals and objectives, estimating the needed resources, forecast the stages of production, anticipate problems in the process, correct the problems, and plan what, when, how much and how to produce (Pérez et al. 2019). It is in planning where a journey towards the planned results and productivity goals are set up with respect to the needed resources.

According to Koskela (1992), there are two main processes in a construction project, design and construction. Then, there are other processes that support and control the main processes such as the construction management process. The latter transforms the design into a production plan, which in the end leads to the product. The processes have a continuous flow of materials, workforce, outputs and activities, that can be either waste (material waste, idle time an spaces, lack of quality, disturbances, etc.) or value (wanted output that adds value to the process and product) (Bølviken et al. 2014).

Lean construction focuses on reducing waste that may exist in any format in the construction process, reducing variability and irregularity to ensure a flow without interruptions, and having material on the site only when needed (Mao and Zhang 2008). 
According to Mao and Zhang (2008), lean has led to positive results, such as higher value, reduced costs and higher productivity. One way that has shown its potentials in increasing productivity is the Last Planner System, a way to plan and control the construction process (Ballard 2000). The idea is based on collaborative visual planning, fomenting communication and commitment between the involved parties, and to enable a pull system flow through the project by stating what needs have to be fulfilled for and by every party before the start of a new activity. By continuous follow-ups of the plan, accompanied by daily huddles (short morning meetings), one control, plan and rearrange production to minimize waste (Fuemana et al. 2013). That is, a way to enable work progress and production flow that focuses on identifying and dealing with waste that obstructs the transformation flow while optimizing execution to get higher productivity.

\section{POSITION TAKEN IN THIS RESEARCH...}

As presented in Figure 1, there is a cloud of factors influencing the inputs, processes and outputs that in turn affect productivity (Hasan et al. 2018). A position taken, to adhere to the propositions in Bernolag (1997) and Pritchard (1995), is that besides measuring productivity as the ratio of output over inputs, assessing productivity also can include measuring or trying to achieve control over the factors related to productivity that affect the inputs, processes or even the output. Thus, an ongoing control of the factors, as well as the inputs and processes to produce outputs, together with measures from the productivity progress of sub-processes or operational activities in site production could support house-builders with indicative means (as Beatham et al. (2004) present) to continuously keep track of, i.e. plan, control and rearrange production, to achieve the predefined productivity, or even productivity improvement goals of production. The consequences of taking this theoretical standpoint is to recognize that practitioners also indirectly measure factors influencing productivity during operations.

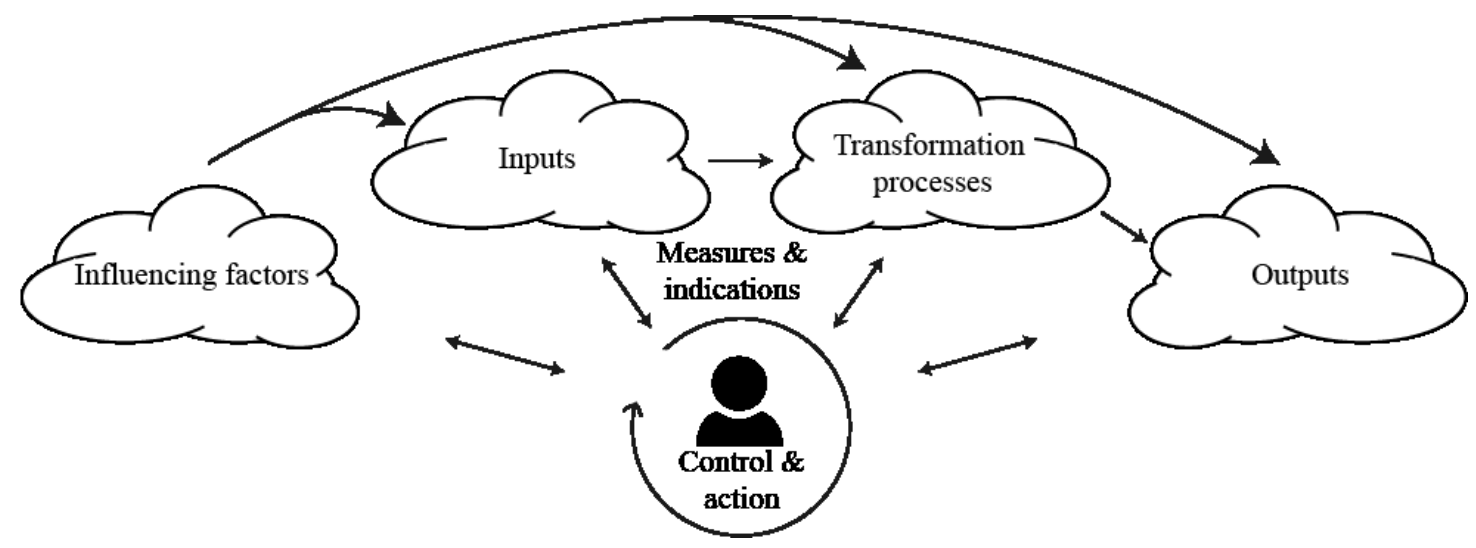

Figure 1: Conceptual map of productivity control in HB

\section{METHOD}

This paper presents the second part from a research that follows an ongoing R\&D study that researchers from Luleå University of Technology are carrying out together with keyrepresentatives from one small and four of the largest Swedish HBCs. The purpose of the study is to identify different productivity measures from the on-site production in HB projects, suggest measures and provide guidance on how the measures can be used to control and improve operations. The first part of the research treated the perceived 
purposes for measuring productivity, views on how to measure, and some challenges associated with measuring productivity for intended purposes (Jimenez et al. 2019).

For the second part, the empirical findings were collected through 13 interviews with IPs from the five HBCs, in different locations in Sweden (northern, eastern, central, western and southern part of the country) to cover eventual geographical differences concerning how companies keep track of productivity. Each interviewee (referred to as IPs) was selected by a senior manager from each company to represent different decision levels, ranging from site managers, to project managers, to business area managers, to cover eventual differences between the organizational levels. The interviews were semistructured covering questions about what productivity is understood as, why measure productivity, how productivity is measured formally (official measures of productivity throughout the organization) and informally (indicative measures and means to assess productivity during production, not reported to the higher levels), and what factors the interviewees consider to affect productivity. Additionally one interview was held with a senior manager from one of the companies to understand better how planning and negotiations with labor unions based on piece rates are carried out before production start.

The data was analyzed first to identify what the companies actually measure, and how they measure and use productivity during production. The analysis included pointing out what factors the IPs consider important for maintaining high productivity in HB projects. The analysis was then presented and discussed in a one-day workshop with five keyrepresentatives from the HBCs. Three researchers took notes of the workshop and the semi-structured discussions were recorded in full.

Finally, acknowledging the view of the perceived purposes of measuring productivity from the first part of the research, the findings were used to discuss literature on productivity, its driving factors in construction, and the use of PIs in processes as a mean to improve operational productivity.

\section{FINDINGS}

\section{PRODUCTIVITY FROM THE COMPANIES POINT OF VIEW}

The site managers' perceive productivity as how much one can complete and how well one performs in transforming resources into an output in a certain amount of time. The output has to fulfill predefined qualities and needs for the next phase, e.g. the next step in the production process or the use of the building. The reason of being aware of productivity is to constantly improve and develop efficiency and effectiveness, with focus on time, but also considering lowering costs in the on-site production process.

"Productivity is about how much one can produce with the right quality during a certain amount of time, including minimizing the use of resources and costs." - IP

The project and business area managers state that productivity also concerns a process that starts from the idea, design and planning phase, continues throughout the on-site production phase, and ends with the fulfilling of the needs and values that the next phase have (which could be the use and management of the building). In addition to what the site managers state, productivity has to do with how little resources (including time, costs, workforce, materials, etc.) one can create the values for the end-users and at the same time, fulfill all the needs that every step in and between the processes have, and thus achieve an ongoing high productivity in the process as a whole. When it comes to the production phase, the companies should have fulfilled the needs for that phase so that a 
planned productivity level can be maintained during production. The idea is to manage resources and create solutions that require less effort (costs, time or resources) to create the values needed in the overall process.

"Productivity is about our competiveness, or ability to get the project" - IP

\section{DEFINING THE PROJECT'S PRODUCTIVITY GOAL}

Firstly, the IPs create a main project plan that includes every plan for each project phase, e.g. a design-, purchase- and production plan. All plans are connected and a predefined productivity is set up in terms of costs and time. On the one hand, there are indicators that relates to the design of a project, e.g. the design of a building. These are applied in the stages before the on-site production, either to make a design with building solutions that would drive profit and productivity in the next stages (production and maintenance), or to evaluate if the companies should accept a project with an already existing design. On the other hand, there are measures of costs and time that the purchase and production planning use to produce a budget and a time plan. With the budget and time plan ready, the companies have set a production plan with a given productivity outcome in terms of time and costs. A plan that the companies assess to be achievable with an acceptable profit and a pace that would allow a production flow without disturbances.

"KPIs and measures are used in the planning of time and costs, together with experience based assessments to set the framework for the project" - IP

Next, the budgeted amount of labor time based on piece rates is negotiated with labor unions. The total amount of man-hours for the given project tasks can then be altered to fit the requirements that the labor unions put on the contractors. With the modified production plan, the contractors finally enter the production phase, with a given productivity outcome, often modified from the first plan, that is meant to be followed and controlled during the construction.

\section{USE OF MEASURES OF PRODUCTIVITY SEEN AS A RESULT}

Every studied company officially measure and report costs and time from production throughout the organizational levels (task, project and organization). The common ground is that accumulated time and costs with respect to the budget and time plan are measured from tasks, added together on the project level, to report the project outcome to the higher organizational levels. Furthermore, during production of large projects, the companies also measure the accumulated times and costs at project level to make prognoses (normally every four months) based on the results achieved so far.

"Bottom line inputs as time and costs are measured vs. the planned time and costs, but knowing what created the margin is difficult and usually not investigated." - IP

At the task level, the companies measure costs to control the result from tasks. Either accumulated costs to control that the outcome stays within the limits of the budget, or to compare offers to cut costs or balance costs between tasks. Accumulated time for tasks is measured to balance the time of tasks and control that nothing exceeds the time schedules. However, if a task took shorter or longer than planned, one normally accepts this without analysing how much time the task could take. Alternatively, if everything is reaching completion within the planned costs and time, one rarely does any further improvements.

"If something took the double in time one usually accept that and think that maybe it takes that time, one seldom think about how long it actually could take" - IP 


\section{CONTROLLING AND ENABLING PRODUCTIVITY DURING OPERATIONS}

There are differences between what is actually measured and reported, and what is assessed to control and act upon the ability to reach the planned costs and times. Different organizations and even projects within the same organizations use measures differently to control productivity depending on the managers. For instance, one organization uses daily huddles to identify and minimize disturbances in the production flow. Another manager develops digitalized statistical diagrams with different measures to control and influence how the project evolves. The studied managers report that the use of digital tools facilitate the control of the project. Digitalization is starting to be used to follow up quality and safety deficiencies and their corrective actions. One of the companies hope to be able to use this to promote the learning of how to avoid or act upon complicated tasks.

The following findings presented are some examples that have been identified of how the interviewees assess, control an enable productivity during production.

\section{Costs and time}

Costs and time are measured in every company, but the use of such measures during operations vary. On task and project level, one of the companies normally measure and adjust accumulated time every two weeks, while another organization updates the main time plan once a week, complemented by daily huddles to identify disturbances in the production flow to then rearrange tasks and minimize time losses on project level.

Regarding costs, one manager measures costs as a result of the tasks to balance costs between tasks with respect to the budget on project level. Another manager puts costs and time in relation to the completed percentage to control and act on the evolution of the costs and time with respect to the work pace and completion of both tasks and the project.

"I measure many things.... costs and time can be set in statistical diagrams to make many different analyses, for example with respect to progress" - IP

\section{Resources (workforce, materials, tools, etc.)}

The number of workers versus the number of ongoing tasks and completion rate is controlled to balance the amount of workers needed over time at task and project level. Another use of this measure is to evaluate the number of workers to avoid congestions.

Two managers state that they use material delivery schedules to control the material flow into the site. By controlling when the material arrives on task level, the team can plan logistics better and rearrange tasks at project level if the material supply fails. With the same purpose, crane schedules are applied to control the material flow into the building. Related to the material, one manager states that they measure the quantity of material needed in BIM and the material waste after tasks to control and optimize the use of material and minimize waste for both the task and project level. Material waste is costly, as every component that is thrown away has attained costs in every step of the process.

"Man-hours are deliberate, together with the work team, under budget resulting in

higher salary for the work staff and reduced total labor cost for the company" - IP

\section{Quality}

The organizations control quality through inspections at task and project level. In large projects, the inspections tend to be ongoing during production and after completion of key tasks. In smaller projects, only at the end. Additionally, the organizations use selfcontrols, which the workers should complete during each task to prevent quality defects. However, results from task defects are seldom incorporated in systematic measurement 
and learning processes at the project and organizational level. The defects are just fixed to meet the quality requirements and the gained knowledge usually stays inside the project.

\section{Work environment}

Another factor that the companies measure is safety. Safety is seen from two perspectives, in the short term it might affect productivity negatively, but in the long term, IPs report to have noticed that it has a positive effect on productivity. Safety is measured systematically as the amount of observations, accidents and absence, to minimize absence on project level. Another measure used regarding safety is the lost time injury frequency, which is the number of injuries that results in absence over one day and four days.

"In short terms it might affect productivity negatively, but we have noticed that working with safety and minimizing accidents pays off in the long run when it comes to both production and productivity" - IP

All managers state that satisfaction and stress levels have an influence on motivation and in turn on productivity. At the same time, satisfaction and the stress levels are understood to give indications of a productive and unhindered production flow. Satisfaction and stress are generally measured using questionnaires at the organizational level, but the managers explain that controlling satisfaction and stress levels at the project level and during the tasks is important, but difficult. The IPs state that to know, they have to get to know the personnel by being on-site, not inside the offices, and have an ongoing communication to build up relationships based on trust and confidence. "Happy and unworried people" is usually a good sign of good workflow and productivity. Another way that the use to check the stress levels and problems is if the working spaces are tidy.

"Satisfied and unworried employees is an effect of doing the things right from start, as

well as satisfaction per se can induce motivation and productivity." - IP

\section{Project culture}

Project culture is a factor that the site managers try to control on the project level to get every participant to work towards the same goal by involving participants from the involved parties in start-up meetings. The higher levels also try to impose the same culture but for every project as an attempt to standardize methods aligned with the companies' visions and goals. The IPs state that based on many years of experience, the organizations know how one should manage the projects to get good results and productivity, what works well in practice and what does not. Through regular meetings with the site managers, the higher levels check how IPs work at the project level, including elements such as standardized methods and routines for procurement, project planning, job planning, meeting intervals, purchase of materials, the use of digitalization and BIM, etc.

"We follow up methods and routines for having meetings, working, procuring, job planning... to build up a culture where everyone is on the same track" - IP

"It is all about trust, honesty, between site manager, workers and subcontractors." - IP

\section{DISCUSSION}

The findings agree with the literature, e.g. Pritchard (1995) and Huang et al. (2009); the understandings and use of productivity differ between contexts, levels and purposes. On the one hand, productivity as presented by Tangen (2005), output/input, is measured as a KPO to report productivity (using time and costs) from production to the higher levels. 
Productivity in this way is also used at task and project level as a KPO in the ways that Beatham et al. (2004) present, to make changes and rearrange production for future tasks. On the other hand, the IPs coincide with the propositions from Pritchard (1995). Productivity also includes assessing many factors that affect the ability to produce, an understanding with common characteristics to the MFP from Crawford and Vogl (2006).

Alike what is presented by Park (2006) and Hasan et al. (2018), the influencing factors, as well as the inputs, transformation processes and output, i.e. systems in HB, are context dependent together with a low degree of standardized systems in the companies. Thus, it is not surprising that the findings indicate that IPs in HB measure and assess different things in different ways when controlling and enabling productivity. Even if productivity is measured as SFP, such as presented by Tangen (2005), its use for improving productivity during production seems to be limited and depends on the user. However, what the IPs do is comparable to the principles of using leading and lagging measures presented by Beatham et al. (2004). Even if many of the measures are not quantitative, the IPs continuously measure many aspects (as in Figure 1) of the influencing factors on productivity, the inputs, processes and output qualitatively. Still, many measures are not formalized nor used systematically, hampering some potentials that measurements have such as knowledge transfer for learning that Lantelme and Formoso (1999) present.

Furthermore, the IPs intend to attain control over the factors presented by Hasan et al. (2018) and Aziz and Hafez (2013), such as the non-availability of materials through controlling the supply of materials, poor communication through e.g. daily huddles, rework through quality control, skill shortening through minimizing absence, and project culture through different meetings (e.g. collaborative start-up and job planning meetings) together with intentions to standardize methods in the organizations; much alike what Koskela (2000) and Ballard (2000) propose. Adhering to the proposition from Pritchard (1995), the IPs indirectly would assess and thus enable for productivity by these means.

The findings indicate that the studied companies take influences from the principles of Lean Construction and relates the use to planning and production control inspired by LPS from Ballard (2000). The focus on elimination of waste, creating transformation flows without interruptions in and between processes, as well as creating value for each production step and client (similar to what Koskela (2000) presents), is something that the IPs consider to be part of productivity. However, project managerial actions are not productivity measures per se (e.g. daily huddles, job planning and LPS), but ways that IPs apply to continuously keep track of and enable for productivity (usually qualitatively).

Formalizing systematic measurements with lagging and leading measures throughout the organizational levels such as presented by Beatham et al. (2004) could benefit the studied HBCs. Benefits such as enhancing the understanding of the system as a whole by increasing transparency in and between processes (like planning and production), as well as helping employees to make decentralized decisions still aligned with the objectives and strategies of the organization, such as presented by Koskela (2000) and Lantelme and Formoso (1999). What the findings indicate is that IPs in HB somehow measure and assess many influencing factors of productivity, together with controlling and assessing aspects related to the inputs, transformation process and the output driven by a predefined productivity. Hence, there is a base for using formalized systematic measurements to measure productivity in ways that would allow the HBCs to keep track of productivity, i.e., plan, control and rearrange production to maintain or improve the productivity ratio, including continuously assessing and acting on the influencing factors, inputs, transformation processes and the output, such as presented in Figure 1. 


\section{CONCLUSIONS}

In this paper, productivity is put in relation to assessing production, or the ability to produce, and studied in one small and four of the largest Swedish HBCs. In the companies, productivity can be and is measured, judged and used in different ways, and there are many elements connected systematically that need to be assessed during the journey towards an output to maintain or even improve productivity as such.

Results indicate that there is a base for a systematic use of measures together with qualitative assessments that could benefit a long-term productivity development. Further investigation is needed to better understand how measurements and routines should be designed to be adopted by the companies, but also to connect e.g. design, planning and production to optimize the total productivity of the system seen as a whole.

Moreover, measurements can help organizations to better understand the HB system and operations through increased transparency. To maximize the potential, and related to the design of measurements and routines, further studies will be carried out to better understand how measures can aid in fomenting organizational learning in HB.

Digitalization and standardization show potential together with attempts that IPs perform in using technology to collect and analyze data that can be used to increase productivity. Still, further research is needed to better understand how this can be carried out and adopted by the organizations, which will be investigated in future research.

Lastly, challenges tied to the varying nature of $\mathrm{HB}$ projects complicate the comparison of measures between projects. How to cope with the differences should be studied further.

\section{ACKNOWLEDGEMENTS}

The Construction Industry's Organization for Research and Development, and the five studied HBCs are gratefully acknowledged for their financial support and time.

\section{REFERENCES}

Alby, V. (1994). "Productivity: measurement and management." AACE, MAT41-47.

Allmon, E., Haas, C. T., Borcherding, J. D. \& Goodrum, P. M. (2000). "U.S. construction Labor Productivity Trends, 1970-1998." J. Con. Eng. Manag., 126(2) 97-104.

Aziz, R. F. and Hafez, S. M. (2013). "Applying lean thinking in construction and performance improvement." Alexandria Engineering Journal, 52(4) 679-695.

Ballard, H. (2000). "The last planner system of production control." PhD Diss., School of Civil engineering, University of Birmingham, UK.

Barbosa, F., Woetzel, J., Mischkle, J., Ribeirinho, M., Sridhar, M., Parsons, M., Bertam, N. and Brown, S. (2017). "Reinventing construction through a productivity revolution,” Technical Report, McKinsey Global Institute.

Beatham, S., Anuba, C. and Thorpe, T. (2004). "KPIs: a critical appraisal of their use in construction." Benchmarking: An International Journal, 11(1) 93-117.

Bernolak, I. (1997). "Effective measurement and successful elements of company productivity: The basis of competitiveness and world prosperity." Int. J. Prod. Econ., 52(1-2) 203-213.

Bølviken, T., Rooke, J. and Koskela, L. (2014). "The wastes of production in construction - a TFV based taxonomy." Proc. 22 ${ }^{\text {nd }}$ Ann. Conf. Int. Group for Lean Construction, Oslo, Norway, pp. 811-822.

Choy, E. and Ruwanpura, J. Y. (2006). "Predecting construction productivity using situation-based simulation models." Can. J. Civil Engineering, 33(12) 1585-1600. 
Crawford, P. and Vogl, B. (2006). "Measuring productivity in the construction industry." Building Research \& Information, 34(3) 208-219.

Dolage, D. A. R. and Chan, P. (2013). "Productivity in Construction - A Critical Review of Research." Engineer, 46(4) 31-42.

Egan, J. (1998). "Rethinking construction”. Technical Report, Crown, London, UK.

Fuemana, J., Puolitaival, T. and Davies, K. (2013). "Last Planner System - a step towards improving the productivity of New Zealand construction." Proc. $21^{\text {st }}$ Ann. Conf. Int. Group for Lean Construction, Fortaleza, Brazil, pp. 679-688.

Haponava, T. and Al-Jibouri, S. (2009). "Identifying key performance indicators for use in control of pre-project stage process in construction." International Journal of Productivity and Performance Management, 58(2) 160-173.

Hasan, A., Baroudi, B., Elmualim, A. and Rameezdeen, R. (2018). "Factors affecting construction productivity: a 30 year systematic review." Engineering, Construction and Architectural Management, 25(7) 916-937.

Huang, A. L., Chapman, R. E. and Butry, D. T. (2009). Metrics and Tools for Measuring Construction Productivity: Technical and Empirical Considerations. Gaithersburg, MD: National Institute of Standards and Technology.

Jimenez, A., Engström, S. and Stehn, L. (2019). "Why (Not) Measuring Productivity in House-building Companies?" Proc. 35 th Ann. ARCOM Conf., Leeds, UK, pp. 194-203.

Koskela, L. (1992). "Application of the new production philosophy to construction" Technical Report, Center for Integrated Fac. Eng., Stanford Univ., Stanford, US.

Koskela, L. (2000). "An exloration towards a production theory and its application to construction." PhD Diss., Technical Research Center of Finland, Espoo.

Lantelme, E. and Formoso, C. T. (1999). "Improving performance through measurement: the application of lean production and organisational learning principles." Proc. $8^{\text {th }}$ Ann. Conf. Int. Group for Lean Construction, Sussex University, Brighton, UK.

Mao, X. and Zhang, X. (2008). "Construction Process Reengineering by Integrating Lean Principles and Computer Simulation Techniques.” J. Con. Eng. Man., 134(5) 371-381.

Motwani, J., Kumar, A. and Novakoski, M. (1995). "Measuring construction productivity: a practical approach." Work Study, 44(8) 18-20.

Park, H.-S. (2006). "Conceptual Framework of Construction Productivity Estimation." KSCE Journal of Civil Engineering, 10(5) 331-317.

Pérez, M. A. Á., Severino, M. S. and Armiñana, E. P. (2019). "An improvement in construction planning: Last Planner System ${ }^{\circledR}$.” Building \& Management, 3(2) 60-70.

Pritchard, R. D. (1995). "Productivity Measurement and Improvement - Organizational Case Studies. ” Praeger, London, UK.

Royal Society of Arts, Manufactures and Commerce (RSA) (1995). "Tomorrow's Company: the role of business in a changing world," Tech. Rep., RSA, London, UK.

Tangen, S. (2005). "Demystifying productivity and performance." International Journal of Productivity and Performance Management, 54(1) 34-46.

Vogl, B. and Abdel-Wahab, M. (2015). "Measuring the Construction Industry's Productivity Performance: Critique of International Productivity Comparisons at Industry Level." J. of Constr. Eng. Manage., 141(4) 04014085.

Zou, J. and Zillante, G. (2005). "Project culture within construction projects: a literature review." Proc. $13^{\text {th }}$ Ann. Conf. Int. Group for Lean Construction, Sydney, Australia, pp. 353-361. 